\title{
Do Theories of the Glass Transition, in which the Structural Relaxation Time Does Not Define the Dispersion of the Structural Relaxation, Need Revision?
}

\author{
K. L. Ngai, ${ }^{*, \dagger}$ R. Casalini, ${ }^{\dagger, \star}$ S. Capaccioli,,${ }^{\S, l}$ M. Paluch,,${ }^{\perp}$ and C. M. Roland ${ }^{\dagger}$ \\ Naval Research Laboratory, Washington D.C. 20375-5320, Chemistry Department, George Mason University, \\ Fairfax, Virginia, Dipartimento di Fisica and INFM, Università di Pisa, Via Buonarroti 2, I-56127, Pisa, Italy, \\ INFM-CNR-CRS SOFT, Piazzale Aldo Moro 2, I-00185 Roma, Italy, and Institute of Physics, Silesian \\ University, ul. Uniwersytecka 4, 40-007 Katowice, Poland
}

Received: June 24, 2005; In Final Form: August 11, 2005

\begin{abstract}
Upon decreasing temperature or increasing pressure, a noncrystallizing liquid will vitrify; that is, the structural relaxation time, $\tau_{\alpha}$, becomes so long that the system cannot attain an equilibrium configuration in the available time. Theories, including the well-known free volume and configurational entropy models, explain the glass transition by invoking a single quantity that governs the structural relaxation time. The dispersion of the structural relaxation (i.e., the structural relaxation function) is either not addressed or is derived as a parallel consequence (or afterthought) and thus is independent of $\tau_{\alpha}$. In these models the time dependence of the relaxation bears no fundamental relationship to the value of $\tau_{\alpha}$ or other dynamic properties. Such approaches appear to be incompatible with a general experimental fact recently discovered in glass-formers: for a given material at a fixed value of $\tau_{\alpha}$, the dispersion is constant, independent of thermodynamic conditions ( $T$ and $P)$; that is, the shape of the $\alpha$-relaxation function depends only on the relaxation time. If derived independently of $\tau_{\alpha}$, it is an unlikely result that the dispersion of the structural relaxation would be uniquely defined by $\tau_{\alpha}$.
\end{abstract}

\section{Introduction}

Vitrification, the dramatic slowing down of kinetic processes such as molecular reorientation, is a general phenomenon found in organic, inorganic, metallic, and polymeric materials. On decreasing temperature $T$ or increasing pressure $P$, the structural relaxation time $\tau_{\alpha}$ becomes long and eventually the liquid cannot reach its equilibrium configuration. The importance of vitrification and glassy behavior in science and technology has inspired continued research efforts over many decades, with the goal of understanding the underlying dynamics and ascertaining the governing factors therein. Those factors that have been identified are incorporated into present-day theories and models of the glass transition. The most notable dynamic property (and the immediate cause of vitrification) is the divergence of $\tau_{\alpha}$ with decreasing temperature at constant pressure. This temperature dependence can be represented in the vicinity of the glass transition by the Vogel-Fulcher-Tammann-Hesse (VFTH) equation $^{1-3}$ or the equivalent Williams-Landel-Ferry (WLF) equation. ${ }^{4}$ The factor governing the divergence of $\tau_{\alpha}$ was identified as unoccupied volume in the free volume model, ${ }^{4,5}$ and configurational entropy in the Adam and Gibbs ${ }^{6}$ model.

\footnotetext{
* Corresponding author.

George Mason University.

† Università di Pisa.

$\S$ INFM-CNR-CRS SOFT.

"Università di Roma "La Sapienza".

$\perp$ Silesian University.
}

An increase of pressure, similar to a temperature decrease, reduces both the unoccupied volume and the configurational entropy, thus slowing down structural relaxation. The free volume model has been extended to consider the effect of hydrostatic pressure, ${ }^{4,7}$ and an extension of the Adam-Gibbs model for elevated pressures has been proposed. ${ }^{8}$ More sophisticated offshoots of these two celebrated models have appeared but are based essentially on the same controlling factors.

Despite differences in the physics underlying the various theories and models of the glass transition, one trait common to all is that the dispersion (time or frequency dependence) of the structural relaxation bears no relation to the structural relaxation time and does not govern the dynamic properties. Instead, the dispersion is either not considered or is derived as a separate consequence of the model based on other factors, possibly entailing additional assumptions. Consequently, the dispersion and $\tau_{\alpha}$ are obtained independently as separate and unrelated predictions. Certainly $\tau_{\alpha}$ can be constant for different combinations of temperature and pressure because of compensating effects on the molecular mobility, even though the specific volume, configurational entropy, and static structure factor may change. However, if the dispersion of the structural relaxation is derived independently of $\tau_{\alpha}$, it is not expected to be constant for these same combinations of $T$ and $P$ since the two quantities do not necessarily have the same dependence on volume, entropy, static structure factor, high-frequency shear modulus, etc.

(C) 2005 American Chemical Society 
In this paper we present representative experimental results for various glass formers to show that the dispersion of the structural relaxation remains unchanged for widely different combinations of temperature and pressure, provided $\tau_{\alpha}$ is constant. This apparently general property implies that the dispersion of the structural relaxation is defined by $\tau_{\alpha}$, or at least $\tau_{\alpha}$ and the dispersion have to be coupled predictions of any viable theoretical interpretation. If the dispersion of the structural relaxation is derived independently of $\tau_{\alpha}$, it is unlikely to be a unique function of $\tau_{\alpha}$. This observation and the conclusions drawn from it have important consequences for studies of the glass transition and the dynamic properties of glass-forming materials. Newly analyzed experimental data, along with some previously published results, are presented herein to support these conclusions.

\section{Invariance of the $\alpha$-Dispersion to Different Combinations of $\boldsymbol{T}$ and $\boldsymbol{P}$ at Constant $\tau_{\alpha}$}

Due largely to experimental convenience, studies of molecular dynamics have focused on the effect of temperature, wherein isobaric measurements of relaxation times and viscosities are carried out as a function of $T$. Recently, pressure has been employed as an experimental variable, with the resulting data leading to discovery of new facts having important insights into the dynamics of glass-forming liquids and polymers. Some of these new findings concerning the dynamic properties are widely applicable and have immediate impact on understanding the glass transition. One example is the dielectric loss spectra measured at atmospheric and elevated pressures for a large number of glass-formers. ${ }^{9-36}$ A given value of the structural relaxation time $\tau_{\alpha}$ or frequency $v_{\alpha}$ at ambient pressure can be maintained upon increases in pressure $P$ by raising the temperature. Various combinations of $P$ and $T$ can be chosen for which the $\alpha$-loss peak frequency $v_{\alpha}$ will remain the same. In cases where the height of the $\alpha$-loss peak, $\epsilon_{\max }^{\prime \prime}$, changes, the dispersions at a fixed $v_{\alpha}$ are compared after the amplitude of the dielectric loss $\epsilon^{\prime}(v)$ has been normalized by $\epsilon_{\max }^{\prime}$. Remarkably, for the same $\alpha$-loss peak frequency there is no change in the frequency dispersion of the $\alpha$-loss peak with varying pressure and temperature. In other words, for a given material at a fixed value of $\tau_{\alpha}$, the $\alpha$-relaxation function is constant, independent of thermodynamic conditions (temperature and pressure). Stated differently, temperature-pressure superpositioning works for the dispersion of the structural $\alpha$-relaxation at constant $\tau_{\alpha}$. Lack of superposition may occur at frequencies sufficiently higher than $v_{\alpha}$. Such deviations are attributed to contributions to the dielectric loss from secondary relaxations (which may not be resolved from the primary $\alpha$-peak), whose relaxation time and dielectric strength do not have the same $P$ and $T$ dependences as the $\alpha$-relaxation.

To demonstrate convincingly this general experimental fact, we use newly analyzed experimental data and provide additional data on previously investigated glass formers. We present dielectric relaxation loss curves measured at different $P$ and $T$ combinations that have more than one value of $\nu_{\alpha}$ or $\tau_{\alpha}$ (and thus different breadths for the dispersion ${ }^{37}$ ). These results show that for a given material, the invariance of the structural relaxation function for fixed $\tau_{\alpha}$ is valid for a broad range of $\tau_{\alpha}$. The materials investigated herein include molecular glass formers (Figure 1) and amorphous polymers (Figure 2) of diverse chemical structure; all show the property of temperature-pressure superpositioning of the $\alpha$-dispersion at constant $\tau_{\alpha}$

\section{Results}

Molecular Glass Formers. We first consider small-molecule, glass-forming liquids that have narrow dispersions of the $\alpha$-relaxation and an excess wing on the high-frequency flank, but otherwise no other resolved secondary relaxations in their dielectric spectra. There are experimental results ${ }^{38-42}$ indicating that the excess wing is an unresolved Johari-Goldstein secondary relaxation. ${ }^{43-46}$ These materials include cresolphthaleindimethyl ether (KDE), ${ }^{9}$ phenylphthalein-dimethyl ether (PDE), ${ }^{10}$ propylene carbonate (PC), ${ }^{11}$ chlorinated biphenyl (PCB62), ${ }^{12}$ phenyl salicylate (salol), ${ }^{13} 3,3^{\prime}, 4,4^{\prime}$-benzophenonetetracarboxylic dianhydride (BPTCDaH), ${ }^{14}$ and 1,1'-di(4-methoxy-5-methylphenyl)cyclohexane (BMMPC). ${ }^{15}$ For these materials, due to the unresolved secondary relaxation, a strong dependence of the shape of the dispersion on $T$ and $P$ (with $\tau_{\alpha}$ varying) is especially evident; see for example refs 9 and 12 . The fact that at a fixed value of $\tau_{\alpha}$ the dispersion of the $\alpha$-relaxation is constant, independent of $T$ and $P$, is demonstrated in Figure 1a for $\mathrm{KDE}$, Figure $1 \mathrm{~b}$ for $\mathrm{PC}$, and Figure $1 \mathrm{c}$ for PCB62 (experimental details of these measurements can be found respectively in refs 9,11 , and 12). In each figure, previously unpublished data are used to show that this property holds for more than one value of $\tau_{\alpha}$. The same results are found (but not shown herein) for PDE, BPTCDaH, BMMPC, and salol.

There are molecular glass formers that have a resolved secondary relaxation whose peak frequency is pressure independent; these are not Johari-Goldstein (JG) processes (according to the definition given in ref 42). The slower JG relaxation is not resolved from the $\alpha$-relaxation in the equilibrium liquid state, but in some cases it can be observed as a distinct peak in the glassy state. Such liquids include 1,1'-bis(p-methoxyphenyl)cyclohexane (BMPC), ${ }^{16}$ diethyl phthalate, (DEP), ${ }^{17}$ di- $n$-butyl phthalate (DBP),${ }^{18}$ diisobutyl phthalate (DiBP),${ }^{18}$ di-isooctal phthalate (DiOP),${ }^{19}$ decahydroisoquinoline (DHIQ), ${ }^{20}$ dipropyleneglycol dibenzoate (DPGDB), ${ }^{21}$ benzoinisobutyl ether (BIBE), ${ }^{22}$ the epoxy compounds including diglycidyl ether of bisphenol A (EPON828), ${ }^{23} 4,4^{\prime}$-methylenebis( $N, N$-diglycidylaniline) (MBDGA),${ }^{24}$ bisphenol A-propoxylate(1 PO/phenol)diglycidyl ether) (1PODGE), ${ }^{25} \mathrm{~N}, N$-diglycidyl4-glycidyloxyaniline (DGGOA), ${ }^{26}$ and $N, N$-diglycidylaniline (DGA) ${ }^{26}$ For all members of this class of glass formers, a constant dispersion is associated with a fixed value of $\tau_{\alpha}$, independent of thermodynamic conditions ( $T$ and $P$ ). We show this (for more than one value of $\tau_{\alpha}$ ) with previously unpublished data in Figure 1d for DiBP, Figure 1e for DPGDB, and Figure 1f for BIBE. The experimental details for these can be found, respectively in refs 18,21 , and 22 . The same property holds also for BMPC, DiBP, DEP, DiOP, DHIQ, EPON828, 1PODGE, MBDGA, DGGOA, and DGA (results not shown).

Earlier dielectric studies under elevated pressure ${ }^{47-51}$ found temperature-pressure superpositioning at constant $\tau_{\alpha}$ for a few molecular glass formers, including ortho-terphenyl (OTP), di(2-ethylhexyl) phthalate, tricresyl phosphate, polyphenyl ether, and a naphthenic mineral oil; however, the temperature and pressure ranges were not as wide as herein.

Amorphous Polymers. Dielectric relaxation measurements under pressure have been carried out on several amorphous polymers and for all cases the dispersion of the local segmental relaxation (i.e., the $\alpha$-relaxation) conforms to temperaturepressure superpositioning at constant $\tau_{\alpha}$. These polymers include polyvinylmethyl ether (PVME), ${ }^{27}$ poly(vinyl acetate) (PVAc), ${ }^{28}$ poly(ethylene-co-vinyl acetate) (EVA, having $70 \mathrm{wt} \%$ vinyl acetate), ${ }^{29}$ polymethylphenylsiloxane (PMPS) ${ }^{30}$ poly(methyltolylsiloxane) (PMTS), ${ }^{31}$ polyvinylethylene (PVE, also referred 

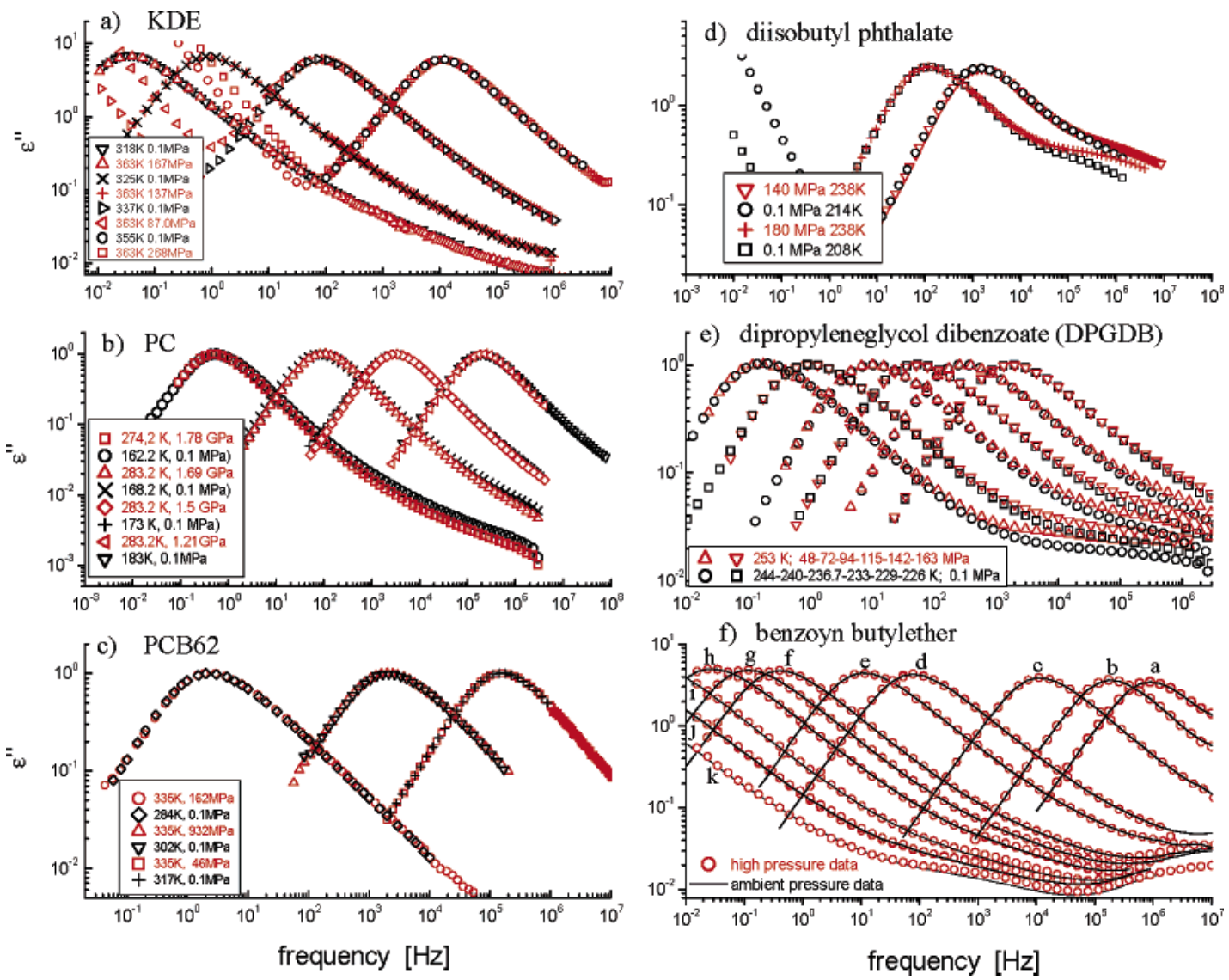

Figure 1. Dielectric loss data at various combinations of temperature and pressure (as indicated) to demonstrate the invariance of the dispersion of the $\alpha$-relaxation at constant $\alpha$-loss peak frequency $v_{\alpha}$ or equivalently at constant $\alpha$-relaxation time $\tau_{\mathrm{a}}$. (a) Cresolphthalein-dimethylether (KDE). (b) Propylene carbonate (PC) (loss normalized to the value of the maximum of the $\alpha$-loss peak measured at ambient pressure). The two ambient pressure spectra at higher frequency are from ref 38. (c) Chlorinated biphenyl (PCB62). (d) Diisobutyl phthalate (DiBP). (e) Dielectric loss of dipropyleneglycol dibenzoate (DPGDB) normalized to the value of the maximum of the $\alpha$-loss peak. The d.c. conductivity contribution has been subtracted. Red triangles are isothermal measurements at $T=253 \mathrm{~K}$ and $P=48,72,94,115,142,163 \mathrm{MPa}$ (from right to left). Black symbols are isobaric measurements made at $P=0.1 \mathrm{MPa}$ and $T=244,240,236.7,233,229,226 \mathrm{~K}$ (from right to left). The spectrum at $T=$ $226 \mathrm{~K}$ has been shifted along $x$-axis by multiplying frequency by a factor 1.3. (f) Dielectric loss of benzoyn butyl ether (BIBE) at different $T$ and $P$. The d.c. conductivity contribution has been subtracted. Spectra obtained at higher $P$ are normalized to the value of the maximum of the loss peak obtained at the same frequency at atmospheric pressure. From right to left: black lines are atmospheric pressure data at $T(\mathrm{~K})=271$ (a), 263 (b), 253 (c), 240 (d), 236 (e), 230 (f), 228 (g), 226 (h), 223 (i), 220.5 (j), 218 (k) K. Symbols are high-pressure data: $T=278.5 \mathrm{~K}$ and $P=32$ (a), 65 (b), 118 (c), 204 (d), 225 (e), 320 (h), 370 (j), 396 (k) MPa; $T=288.2 \mathrm{~K}$ and $P=350$ (f), 370 (g), 423 (i), 450 (j) MPa, and $T=298 \mathrm{~K}$ and $P=330(\mathrm{~d}), 467$ (h) MPa.

to as 1,2-polybutadiene), ${ }^{32}$ poly(phenyl glycidyl ether)-coformaldehyde (PPGE) ${ }^{33}$ 1,4-polyisoprene (PI) ${ }^{34}$ poly(propylene glycol) (molecular weight: $4000 \mathrm{Da})$ PPG-4000, ${ }^{35}$ and poly(oxybutylene) POB. ${ }^{36}$ Including some previously unpublished data, constant dispersions at a fixed value of $\tau_{\alpha}$ independent of thermodynamic conditions ( $T$ and $P$ ) are shown for more than one $\tau_{\alpha}$ in Figure 2a for PVAc, in Figure 2b for PMTS, in Figure $2 \mathrm{c}$ for PPGE, and in Figure 2d for POB. Experimental details for these measurements can be found in refs $28,31,33$, and 36 , respectively. Note that for $\mathrm{POB}$ there is a dielectrically active normal mode at lower frequencies, which has different $P$ and $T$ dependences than the local segmental mode. The $T-P$ superposition at fixed $\tau_{\alpha}$ holds also for PVME, EVA, PMPS, PVE, and PPG-4000 (data not shown).

Hydrogen bonded networks or clusters, if present, are modified at elevated pressure and temperature, changing the physical structure of the glass-former. This occurs, for example, in glycerol, ${ }^{52}$ propylene glycol dimer $(2 \mathrm{PG}),{ }^{40}$ and m-fluoraniline. ${ }^{53}$ These hydrogen bonded glass formers do not obey temperature-pressure superpositioning at constant $\tau_{\alpha}$, since the physical structure of the material changes at elevated pressure/ elevated temperature. ${ }^{54}$

\section{Discussion}

We now discuss the impact of this general property on theories and models of the glass transition. The primary concern of most theories is to explain the temperature and pressure dependences of the structural relaxation time. The dispersion of the structural relaxation is either not addressed or else is drawn separately from additional considerations not involved in arriving at $\tau_{\alpha}$. For example, the original free volume models $s^{4,5}$ and the Adam-Gibbs model ${ }^{6}$ treat the variation of relaxation times with $T$ but do not predict the distribution of molecular relaxation rates. Additional input such as a specific fluctuation of some parameter must be introduced to generate a distribution of relaxation times. It is not difficult for any of these theories to find combinations of $T$ and $P$ such that the predicted $\tau_{\alpha}(T, P)$ is constant. However, it is unlikely that the same combinations will also yield an invariant dispersion. For a single glass former it may be possible to introduce additional assumptions to force both $\tau_{\alpha}$ and the dispersion to be simultaneously constant. However, this would not be a worthwhile undertaking since $\tau_{\alpha}$ and the dispersion are simultaneously constant for so many glass-formers, with different physical and chemical structures, and greatly different sensitivities to temperature and density. ${ }^{55,56}$ 

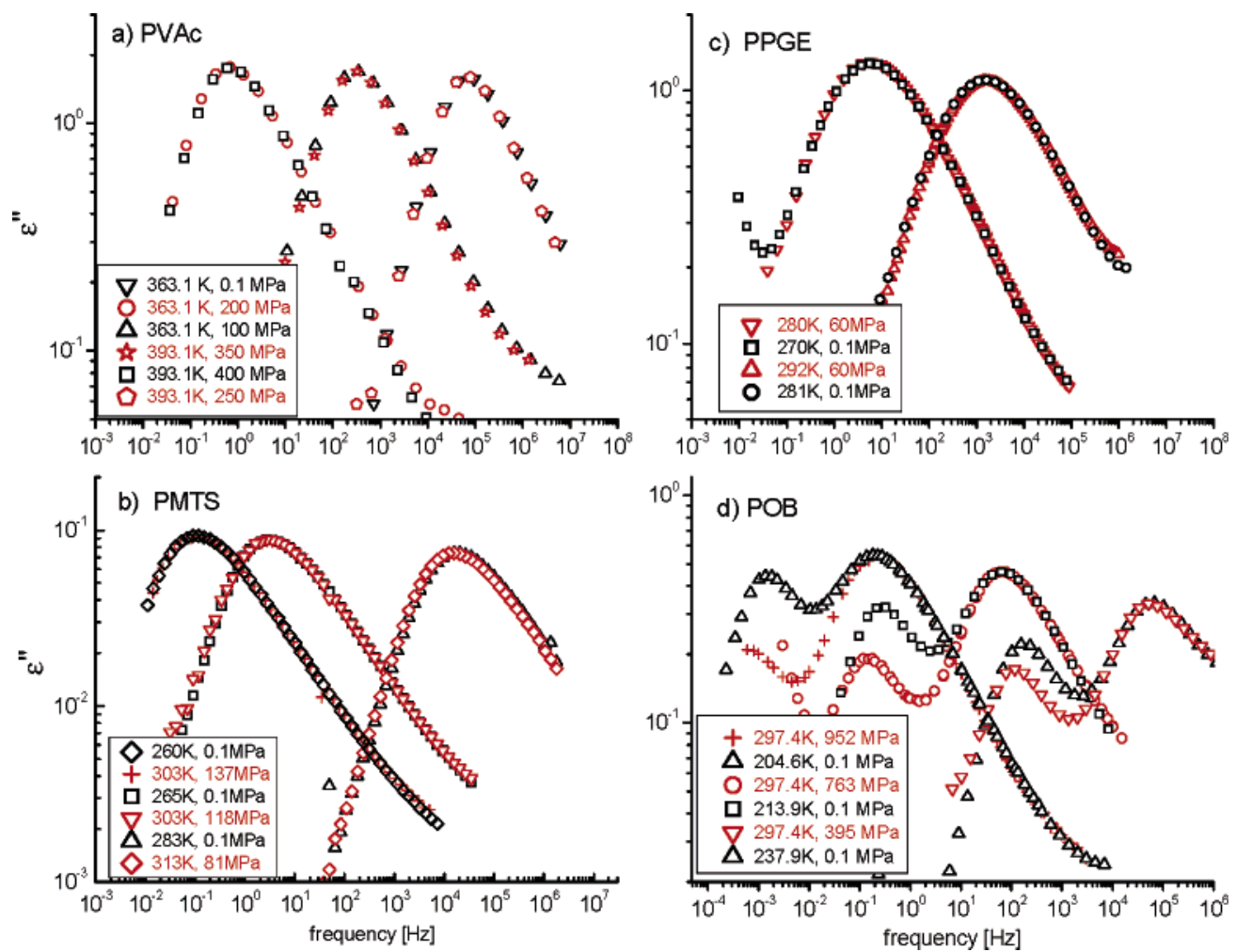

Figure 2. Dielectric loss data at various combinations of temperature and pressure (as indicated) to demonstrate the invariance of the dispersion of the $\alpha$-relaxation at constant $\alpha$-loss peak frequency $v_{\alpha}$ or equivalently at constant $\alpha$-relaxation time $\tau_{\alpha}$. (a) Poly(vinyl acetate) (PVAc); (b) poly(methyltolylsiloxane) (PMTS); (c) poly(phenyl glycidyl ether)-co-formaldehyde (PPGE); (d) poly(oxybutylene) (POB). In all cases, spectra obtained at higher $P$ are normalized to the value of the maximum of the loss peak obtained at the same frequency at atmospheric pressure.

More serious revision is necessary to bring the conventional models into consistency with the general experimental property described herein.

As illustrated in some of the figures, the $\alpha$-loss peaks are well represented by the one-sided Fourier transform of the Kohlrausch function, ${ }^{57,58}$

$$
\phi(t)=\exp \left[-\left(t / \tau_{\alpha}\right)^{1-n}\right]
$$

especially the main peak and its low-frequency flank. ${ }^{37}$ The fractional exponent, $n$, can be used to characterize the dispersion of the $\alpha$-relaxation. Thus, the experimental fact of constant dispersion of the $\alpha$-relaxation at constant $\tau_{\alpha}$ can be restated as the invariance of the fractional exponent or the Kohlrausch exponent, $(1-n)$, to different combinations of $T$ and $P$ that hold $\tau_{\alpha}$ constant. The coupling model $(\mathrm{CM})^{59-63}$ is consistent with this experimental observation. In the $\mathrm{CM}$, at any $T$ and $P$, it is the heterogeneous many-molecule dynamics that slow the primitive relaxation rate, $1 / \tau_{0}(T, P)$, and give rise to the Kohlrausch correlation function. The relaxation time $\tau_{\alpha}(T, P)$ and the fractional exponent $n$ of the Kohlrausch function are linked by the relation $\tau_{\alpha}(T, P)=\left[t_{c}{ }^{-n} \tau_{0}(T, P)\right]^{1 /(1-n)}$. From this defining equation of the $\mathrm{CM}$, it can be shown that $\tau_{\alpha}$ and $n$ are co-invariants for different combinations of $T$ and $P$.

Not only does $\tau_{\alpha}$ uniquely define the dispersion, as shown herein, but also many properties of $\tau_{\alpha}$ are governed by the dispersion or the fractional exponent $n$. Examples of these properties are described in a review $;{ }^{64}$ here a few are mentioned.

(i) The "fragility" index, $m \equiv d \log { }_{10} \tau_{\alpha} /\left.d\left(T_{g} / T\right)\right|_{T g / T=1}$, at ambient pressure increases with $n \cdot{ }^{64,65}$ However, there are wellknown examples of violation of this correlation. This is because the temperature dependence of $\tau_{\alpha}$ is governed not only by $n$ (i.e., the breadth of the dispersion) but also by the specific volume, $V$, and entropy, $S$. Hence, $m$ is not a fundamental quantity. Chemically different glass-formers can have widely different dependence of $\tau_{\alpha}$ on $V$ and $S,{ }^{55}$ causing a breakdown of the correlation between $m$ and $n$. Also for a given glassformer the correlation is found to break down under elevated pressure. ${ }^{67}$ In general $m$ decreases with $P .{ }^{68}$ Since $n$ or the dispersion is constant at $\tau_{\alpha}=10^{2} \mathrm{~s}$ (a time customarily used to define $T_{\mathrm{g}}$ for any pressure), the fact that $m$ decreases with $P$ means that the correlation between $m$ and $n$ necessarily fails.

(ii) In quasielastic neutron scattering experiments, the dependence of $\tau_{\alpha}$ on the scattering vector $Q$ is given by $Q^{2 /(1-n)}$. ${ }^{4,69}$

(iii) The separation, $\left[\log \left(\tau_{\alpha}\right)-\log \left(\tau_{\mathrm{JG}}\right)\right]$, of $\tau_{\alpha}$ from the Johari-Goldstein relaxation time $\tau_{\mathrm{JG}}$ at a fixed value of $\tau_{\alpha}$ is proportional to $n$. $^{42,62,63,70}$

(iv) The observed crossover at some temperature $T_{\mathrm{B}}$ above $T_{\mathrm{g}}$ of the temperature dependence of $\tau_{\alpha}$ from one VFTH equation to another,,$^{70-73}$ the apparent onset of bifurcation of $\tau_{\mathrm{JG}}$ from $\tau_{\alpha},{ }^{39}$ and the decoupling of translational diffusion from viscosity ${ }^{75}$ are all related to the small size of $n$ at temperatures above $T_{\mathrm{B}}$ and the more rapid increase of $n$ across $T_{\mathrm{B}}$ on decreasing the temperature.

( $v$ ) The ratio of the two values of $\tau_{\alpha}$ at $T_{\mathrm{g}}$ given by the two VFTH equations used to fit $\tau_{\alpha}$ at temperatures above and below $T_{\mathrm{B}}$ increases with increasing $n .^{74}$

(vi) For polymers, there is also the correlation of the degree of thermorheological complexity with $n$, as exemplified by polystyrene and polyisobutylene. ${ }^{64,76}$

These properties are all consistent with the coupling model. ${ }^{61}$

\section{Conclusion}

A recently discovered, general experimental fact is that for virtually any glass formers at fixed $\tau_{\alpha}$, the shape of the $\alpha$-dispersion is constant, independent of thermodynamic condi- 
tions. If models of the glass transition derive the dispersion of the structural relaxation independently of $\tau_{\alpha}$, it is unlikely that $\tau_{\alpha}$ would uniquely define the dispersion. With the exception of the coupling model, theories and models of the glass transition either do not address the dispersion or derive it independently of $\tau_{\alpha}$; hence, they are in need of revision.

Acknowledgment. The work at NRL was supported by the Office of Naval Research, at the Università di Pisa by I.N.F.M. and by MIUR (Cofin2002), and at Silesian University by the Polish Committee for Scientific Research (KBN) (Project 20052007; No 1PO3B 075 28).

\section{References and Notes}

(1) Vogel, H. Phys. Z. 1921, 222, 645.

(2) Fulcher, G. S. J. Am. Ceram. Soc. 1923, 8, 339

(3) Tammann, V. G.; Hesse, W. Z. Anorg. Allg. Chem. 1926, 156, 245

(4) Ferry, J. D. Viscoelastic Properties of Polymers, 3rd ed.; Wiley: New York, 1980.

(5) Doolittle, A. K.; Doolittle, D. B. J. Appl. Phys. 1957, 28, 901.

(6) Adam, G.; Gibbs, J. H. J. Chem. Phys. 1965, 43, 139

(7) Dlubek, G.; Wawryszczuk, J.; Pionteck, J.; Gowrek, T.; Kaspar, H.; Lochhass, K. H. Macromolecules 2005, 38, 429.

(8) Casalini, R.; Capaccioli, S.; Lucchesi, M.; Rolla, R. A.; Corezzi, S. Phys. Rev. E 2001, 63, 031207.

(9) Paluch, M.; Ngai, K. L.; Hensel-Bielowka, S. J. Chem. Phys. 2001, 114,10872

(10) Casalini, R.; Paluch, M.; Roland, C. M. J. Chem. Phys. 2003, 118 5701. In a recent paper, Kahle, S.; Gapinski, J.; Hinze, G.; Patkowski, A.; Meier, G. J. Chem. Phys. 2005, 122, 074506, a new secondary dielectric loss process was reported, which can be attributed to the $180^{\circ}$ flip of the phenyl rings in PDE.

(11) Pawlus, S.; Casalini, R.; Roland, C. M.; Paluch, M.; Rzoska, S. J.; Ziolo, J. Phys. Rev. E 2004, 70, 061501.

(12) Casalini, R.; Paluch, M.; Fontanella, J. J.; Roland, C. M. J. Chem. Phys. 2003, 117, 4901

(13) Casalini, R.; Paluch, M.; Roland, C. M. J. Phys. Chem. A 2003 $107,2369$.

(14) Johari, J. P.; Whalley, E. Faraday Symp. Chem. Soc. 1972, 6, 23

(15) Casalini, R.; Paluch, M.; Roland, C. M. Phys. Rev. E 2003, 67, 031505 .

(16) Hensel-Bielowka, S.; Ziolo, J.; Paluch, M.; Roland, C. M. J. Chem. Phys. 2002, 117, 2317.

(17) Pawlus, S.; Paluch, M.; Sekula, M.; Ngai, K. L.; Rzoska, S. J.; Ziolo, J. Phys. Rev. E 2003, 68, 021503.

(18) Sekula, M.; Pawlus, S.; Hensel-Bielowka, S.; Ziolo, J.; Paluch, M.; Roland, C. M. J. Phys. Chem. B 2004, 108, 4997.

(19) Ngai, K. L.; Kamiñska, E.; Sekula, M.; Paluch, M., unpublished.

(20) Richert, R.; Duvvuri, K.; Duong, L.-T. J. Chem. Phys. 2003, 118 , 1828. Paluch, M.; Pawlus, S.; Hensel-Bielowka, S.; Kaminska, E.; Prevosto, D.; Capaccioli, S.; Rolla, P. A.; Ngai, K. L. J. Chem. Phys. 2005, 122 , 234506.

(21) Capaccioli, S.; Prevosto, D.; Lucchesi, M.; Rolla, P. A.; Casalini, R.; Ngai, K. L. J. Non-Cryst. Solids, in press.

(22) Capaccioli, S.; Casalini, R.; Prevosto, D., unpublished.

(23) Corezzi, S.; Rolla, P. A.; Paluch, M.; Ziolo, J.; Fioretto, D. Phys.

Rev. E 1999, 60, 4444

(24) Psurek, T.; Ziolo, J.; Paluch, M. Physica A 2004, 331, 353. Casalini, R.; Psurek, T.; Paluch, M.; Roland, C. M. J. Mol. Liq. 2004, 111, 53.

(25) Psurek, T.; Hensel-Bielowka, S.; Ziolo, J.; Paluch, M. J. Chem. Phys. 2002, 116, 9882.

(26) Hensel-Bielowka, S.; Psurek, T.; Ziolo, J.; Paluch, M. Phys. Rev. E 2001, 63, 62301.

(27) Casalini, R.; Roland, C. M. J. Chem. Phys. 2003, 119, 4052.

(28) Heinrich, W.; Stoll, B. Colloid Polym. Sci. 1985, 263, 873. Schönhals, A.; Ngai, K. L., unpublished.

(29) Zhang, S. H.; Casalini, R.; Runt, J.; Roland, C. M. Macromolecules 2003, 36, 9917 0932.

(31) Paluch, M.; Pawlus, S.; Roland, C. M. Macromolecules 2002, 35, 7338.

(32) Roland, C. M.; Casalini, R.; Santangelo, P.; Sekula, M.; Ziolo, J.; Paluch, M. Macromolecules 2003, 36, 4954

(33) Paluch, M.; Hensel-Bielowka, S.; Ziolo, J. Phys. Rev. E 2000, 61, 526.

(34) Roland, C. M.; Casalini, R.; Paluch, M. J. Polym. Sci. Polym. Phys. Ed. 2004, 42, 4313
(35) Roland, C. M.; Psurek, T.; Pawlus, S.; Paluch, M. J. Polym. Sci.: Polym. Phys. 2003, 41, 3047.

(36) Casalini, R.; Roland, C. M. Macromolecules 2005, 38, 1779.

(37) For a given glass-former, the $\alpha$-relaxation dispersion usually (but not always) broadens with decreasing temperature, and it also varies among different glass formers. Studies purporting to find a universal value of the Kohlrausch exponent describing the shape of the $\alpha$-peak [e.g., Dyre, J. C. Phys. Rev. E 2005, 72, 011501] restrict their attention to the high-frequency tail of the dispersion, rather than the central peak and its low-frequency side considered herein.

(38) Schneider, U.; Brand, R.; Lunkenheimer, P.; Loidl, A. Phys. Rev. Lett. 2000, 84, 5560.

(39) Ngai, K. L.; Lunkenheimer, P.; Leon, C.; Schneider, U.; Brand, R.; Loidl, A. J. Chem. Phys. 2001, 115, 1405.

(40) Casalini, R.; Roland, C. M. Phys. Rev. Lett. 2003, 91, 15702. Phys. Rev. B 2004, 69, 094202 .

(41) Svanberg, C.; Bergman, R.; Jacobsson, P. Europhys. Lett. 2003, 64,358

(42) Ngai, K. L.; Paluch, M. J. Chem. Phys. 2004, 120, 857.

(43) Johari, G. P.; Goldstein, M. J. Chem. Phys. 1970, 53, 2372.

(44) Johari, G. P. J. Chem. Phys. 1973, 58, 1766.

(45) Johari, G. P. Ann. N. Y. Acad. Sci. 1976, 279, 117.

(46) Johari, G. P. J. Non-Cryst. Solids 2002, 307-310, 317.

(47) Naoki, M.; Matsushita, M. Bull. Chem. Soc. Jpn. 1983, 56, 2396. 4169.

48) Naoki, M.; Endou, H.; Matsumoto, K. J. Phys. Chem. 1987, 91 ,

(49) Masuko, M.; Suzuki, A.; Hanai, S.; Okabe, H. Jpn. J. Tribol. 1997, $42,455$.

(50) Suzuki, A.; Masuko, M.; Nakayama, T.; Okabe, H. Jpn. J. Tribol. 1997, $42,467$.

(51) Suzuki, A.; Masuko, M.; Nikkuni, T. Tribol. Int. 2000, 33, 107.

(52) Paluch, M.; Casalini, R.; Hensel-Bielowka, S.; Roland, C. M. J. Chem. Phys. 2002, 116, 9839.

(53) Hensel-Bielowka, S.; Paluch, M.; Ngai, K. L. J. Chem. Phys. 2005, 123,014502 .

(54) Roland, C. M.; Casalini, R.; Paluch, M. Chem. Phys. Lett. 2003, $367,259$.

(55) Casalini, R.; Roland, C. M. Phys. Rev. E 2004, 69, 062501.

(56) Casalini, R.; Roland, C. M. Colloid Polym. Sci. 2004, 283, 107.

(57) Kohlrausch, R. Prog. Ann. Phys. 1847, 12(3), 393.

(58) Williams, G.; Watts, D. C. Trans. Faraday Soc. 1970, 66, 80.

(59) Ngai K. L.; Tsang, K. Y.Phys. Rev. E 1999, 60, 4511.

(60) Ngai, K. L.; Rendell, R. W. in Supercooled Liquids, Advances and Novel Applications; Fourkas, J. T., Kivelson, D., Mohanty, U., Nelson, K., Eds.; ACS Symposium Series Vol. 676; American Chemical Society: Washington, DC, 1997; Chapter 4, p 45.

(61) Ngai, K. L. IEEE Trans. Dielectr. Electron. Insul. 2001, 8, 329.

(62) Ngai, K. L. J. Phys.: Condens. Matter 2003, 15, S1107.

(63) Prevosto, D.; Capaccioli, S.; Lucchesi, M.; Rolla, P. A.; Ngai, K. L. J. Chem. Phys. 2004, 120, 4808.

(64) Ngai, K. L. J. Non-Cryst. Solids 2000, 7, 275.

(65) Böhmer, R.; Ngai, K. L.; Angell, C. A.; Plazek, D. J. J. Chem. Phys. 1993, 99, 4201 .

(66) Ngai, K. L.; Roland, C. M. Macromolecules 1993, 26, 6824.

(67) Roland, C. M.; Paluch, M.; Rzoska, S. J. J. Chem. Phys. 2003, $119,12439$.

(68) Casalini, R.; Roland, C. M. Phys. Rev. B 2005, 71, 014210.

(69) For more recent works, see Neelakantan, A.; Maranas, J. K. J. Chem. Phys. 2004, 120, 465. Neelakantan, A.; Maranas, J. K. J. Chem. Phys. 2004, 120, 1617, and references therein.

(70) Ngai, K. L. J. Chem. Phys. 1998, 109, 6982. 6251

(71) Stickel, F.; Fischer, E. W.; Richert, R. J. Chem. Phys. 1995, 102

(72) Casalini, R.; Ngai, K. L.; Roland, C. M. Phys. Rev. B 2003, 68 , 014201

(73) Ngai K. L.; Roland, C. M. Polymer 2002, 43, 567.

(74) Leon, C.; Ngai, K. L. J. Phys. Chem. 1999, 103, 4045. 1887.

75) Ngai, K. L.; Magill, J. H.; Plazek, D. J. J. Chem. Phys. 2001, 112,

(76) Ngai, K. L.; Plazek, D. J. Rubber Chem. Technol. Rubber Rev. $\mathbf{1 9 9 5}, 68,376$ 